\title{
Publisher Correction: A repeated molecular architecture across thalamic pathways
}

James W. Phillips (1), Anton Schulmann, Erina Hara, Johan Winnubst, Chenghao Liu (1), Vera Valakh, Lihua Wang, Brenda C. Shields, Wyatt Korff, Jayaram Chandrashekar, Andrew L. Lemire, Brett Mensh, Joshua T. Dudman (iD, Sacha B. Nelson (1) and Adam W. Hantman (1)

Correction to: Nature Neuroscience https://doi.org/10.1038/s41593-019-0483-3, published online 16 September 2019.

In the version of this article initially published, in Fig. 3c, the nuclei labels from left to right should have been CM, VA, and VL. The error has been corrected in the HTML and PDF versions of the article.
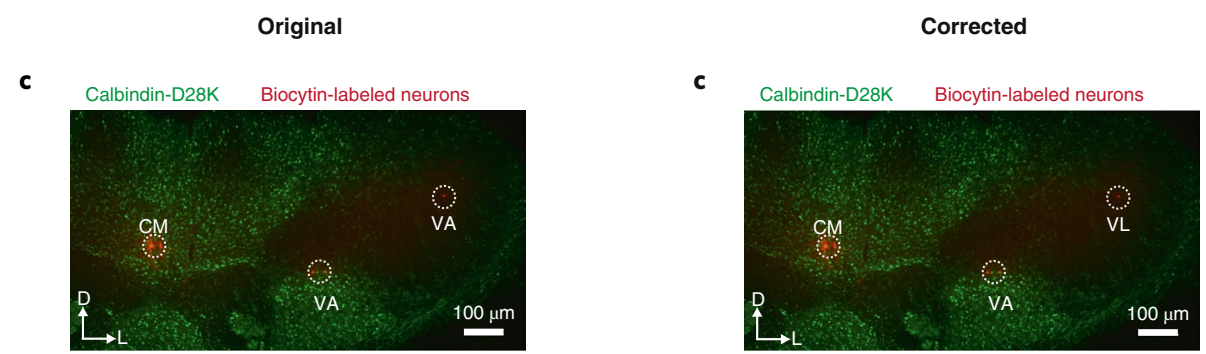

Fig. 3 | Original and Corrected.

Published online: 1 October 2019

https://doi.org/10.1038/s41593-019-0521-1

\section{Publisher Correction: A neural circuit for comorbid depressive symptoms in chronic pain}

Wenjie Zhou, Yan Jin, Qian Meng, Xia Zhu, Tongjian Bai, Yanghua Tian, Yu Mao, Likui Wang, Wen Xie, Hui Zhong, Na Zhang, Min-Hua Luo (D), Wenjuan Tao, Haitao Wang, Jie Li, Juan Li, Ben-Sheng Qiu, Jiang-Ning Zhou, Xiangyao Li $\mathbb{D}$, Han Xu, Kai Wang, Xiaochu Zhang, Yong Liu, Gal Richter-Levin (D), Lin Xu (D) and Zhi Zhang (D)

Correction to: Nature Neuroscience https://doi.org/10.1038/s41593-019-0468-2, published online 26 August 2019.

In the version of this article initially published, author Gal Richter-Levin's surname was misspelled. The error has been corrected in the HTML and PDF versions of the article. 